Bundesgesundheitsbl 2014 · 57:93-102 DOI 10.1007/s00103-013-1869-0

Online publiziert: 20. Dezember 2013

c) Springer-Verlag Berlin Heidelberg 2013
A. Nennecke ${ }^{1} \cdot$ A. Wienecke ${ }^{2} \cdot \mathrm{K}$. Kraywinkel ${ }^{2}$

${ }^{1}$ Hamburgisches Krebsregister, Behörde für Gesundheit und Verbraucherschutz (BGV), Hamburg

2 Zentrum für Krebsregisterdaten, Robert Koch-Institut, Berlin

\title{
Inzidenz und Überleben bei Leukämien in Deutschland nach aktuellen standardisierten Kategorien
}

\begin{tabular}{|ll}
\hline \multicolumn{2}{|l}{ Abkürzungsverzeichnis } \\
\hline$A L L$ & Akute lymphatische Leukämie \\
\hline$A M L$ & Akute myeloische Leukämie \\
\hline CLL & $\begin{array}{l}\text { Chronische lymphatische Leuk- } \\
\text { ämie }\end{array}$ \\
\hline$C M L$ & Chronische myeloische Leukämie \\
\hline$D C O$ & $\begin{array}{l}\text { "death certificate only", ausschließ- } \\
\text { lich über Todesbescheinigungen } \\
\text { im Krebsregister erfasste Fälle }\end{array}$ \\
\hline EKR & $\begin{array}{l}\text { epidemiologische(s) Landeskrebs- } \\
\text { register }\end{array}$ \\
\hline ICD-10 & $\begin{array}{l}\text { Internationale statistische Klassi- } \\
\text { fikation der Krankheiten und ver- } \\
\text { wandten Gesundheitsprobleme, } \\
10 . \text { Revision }\end{array}$ \\
\hline ICD-O-3 & $\begin{array}{l}\text { Internationale Klassifikation der } \\
\text { Krankheiten für die Onkologie, } \\
\text { 3. Revision }\end{array}$ \\
\hline $\begin{array}{l}\text { IR (ASR } \\
\text { Europa) }\end{array}$ & $\begin{array}{l}\text { Inzidenzrate(n), altersstandardi- } \\
\text { siert entsprechend der Europa- } \\
\text { Standardbevölkerung }\end{array}$ \\
\hline o.n.A. & \begin{tabular}{l} 
ohne nähere Angabe \\
\hline WHO
\end{tabular} \begin{tabular}{l} 
Weltgesundheitsorganisation \\
\hline
\end{tabular} \\
\hline
\end{tabular}

\section{Hintergrund}

Der Begriff „Leukämie“, abgeleitet von altgriechisch $\lambda \varepsilon v \kappa o ́ \varsigma$, „weiß“ und aína „Blut“, stammt von Rudolf Virchow, der ihn Mitte des 19. Jahrhunderts angesichts der stark vermehrten weißen Blutkörperchen bei einer bis dahin nicht definierten Erkrankungsart einführte [1]. Otto Naegeli differenzierte bereits um 1890 myeloische und lymphatische Formen, während nach heutigem Verständnis Leukämien eine heterogene Vielzahl von Krebserkrankungsarten des blutbildenden Systems bezeichnen [2]. Sie unterscheiden sich bezüglich Häufigkeit, Ursachen, Behandlungsmöglichkeiten und Heilungsaussichten teils stark voneinander. Leukämien lassen sich nach morphologischen und immunologischen Eigenschaften der Zellen differenzieren, zunehmend auch anhand zytogenetischer und molekularbiologischer Merkmale. Dies ermöglicht eine feine Unterteilung, der die bisher in großen bevölkerungsbezogenen Analysen verwendeten groben Kategorien - akut vs. chronisch und lymphatisch vs. myeloisch - nur begrenzt Rechnung tragen $[3,4]$. Hinzu kommen erhebliche Unterschiede in der Erfassungs- und Kodierungspraxis von Krebsregistern, die zeitliche und räumliche Vergleiche von epidemiologischen Daten zu Leukämien erschweren [5].

Die neueren Gliederungssysteme für hämatologische Erkrankungen, die 2000 von der Weltgesundheitsorganisation (WHO) herausgegebene 3. Revision der Internationalen Klassifikation der Krankheiten für die Onkologie (ICD-O-3) sowie die darauf Bezug nehmenden offiziellen medizinisch-wissenschaftlichen Klassifikationen der WHO (WHO Classification of Tumors of Haematopoietic and Lymphomatic Tissue, sog. „Blue Book" der Hämato-Onkologie) von 2001 und 2008 klassifizieren primär nach Zelllinien und -reife $[6,7,8]$. Zusätzlich verwenden sie morphologische, genotypische, genetische und immunhistochemische Kriterien ebenso wie klinisches Verhalten, um die Entitäten weiter zu differenzieren. Die resultierenden Subkategorien umfassen jedoch z. T. so wenige Er- krankungsfälle, dass bestimmte bevölkerungsbezogene Analysen selbst auf internationaler Ebene problematisch bleiben [9]. In dieser Situation wurde 2005 das von der Europäischen Kommission geförderte HAEMACARE-Projekt initiiert mit dem Ziel, für Inzidenz- und Überlebenszeitanalysen sinnvolle und nützliche standardisierte Gruppierungen hämatoonkologischer Erkrankungen zu definieren [5]. Im Rahmen des Projektes entwickelte eine Expertengruppe von Pathologen, Hämatologen und Klinikern in Kooperation mit europäischen Krebsregistern eine Struktur, die pathophysiologisch und prognostisch ähnliche Erkrankungen zusammenfasst. Ausgehend von den ICD-O-3-Morphologiekodes wird darin zunächst entsprechend den WHOEmpfehlungen zwischen lymphatischen und myeloischen Neubildungen unterschieden. Beide „Familien“ sind in je 5 Hauptgruppen und teils noch weiter in Subgruppen unterteilt [3]. Das HAEMACARE-System baut damit auf den Definitionen der in Krebsregistern gängigen ICD-O-3 auf und ist gleichzeitig kompatibel mit den aktuellen, klinisch relevanten WHO-Klassifikationen. Es ermöglicht eine Verbindung zwischen klinischer Forschung und Public-Health-Informationen. Das von der Arbeitsgruppe konsentierte Ergebnis steht seit 2010 als ein auch über das Internet veröffentlichtes Manual zur Verfügung und wurde bereits für Analysen europäischer Krebsregisterdaten angewendet [10].

In Deutschland sind Neuerkrankungszahlen für Leukämien bei Erwachsenen 
derzeit von einer Reihe epidemiologischer Landeskrebsregister (EKR) über deren interaktive Internetdatenbanken bis einschließlich des Diagnosejahres 2010 abrufbar [11, 12, 13, 14, 15, 16]. Dort finden sich die Diagnosen in der Regel kodiert nach der 10. Revision der Internationalen statistischen Klassifikation der Krankheiten und verwandten Gesundheitsprobleme (ICD-10), für die insbesondere in Bezug auf hämatoonkologische Erkrankungen trotz regelmäßiger Aktualisierungen nur eine begrenzte Anpassung an den aktuellen Stand der Wissenschaft konstatiert wird [17]. 2011 wurden mit einem sog. „major update“ der ICD-10 deutlich verbesserte Anpassungen an die Terminologie der ICD-O-3 und der WHO „Blue Books" erreicht, die jedoch aufgrund fehlender Überleitungsregeln in den vorliegenden Daten der EKR noch nicht berücksichtigt werden konnten [18].

Inzidenzschätzungen für Leukämien in Deutschland insgesamt werden regelmäßig über den interaktiven Krebs-Atlas der Gesellschaft der epidemiologischen Krebsregister in Deutschland e.V. und über die Internetseite des Zentrums für Krebsregisterdaten am Robert KochInstitut veröffentlicht, hier allerdings nur für die grob als „Leukämien“ zusammengefasste Gruppe ICD-10 C91-C95 [19, 20]. Auch die bisher im Rahmen von EKRJahresberichten oder einzelnen wissenschaftlichen Publikationen bekannt gegebenen bevölkerungsbezogenen Überlebensdaten beziehen sich ausschließlich auf die Kategorien der ICD-10 [21, 22]. Ziel der vorliegenden Arbeit ist es, erstmals für Deutschland Zahlen zu Neuerkrankungen und zum Überleben bei Leukämien erwachsener Patienten nach der HAEMACARE-Gruppierung anhand eines Pools mehrerer deutscher Landeskrebsregister darzustellen und zu bewerten.

\section{Methoden}

\section{Daten}

Der verwendete Datensatz wurde aus den nach $₫ 3$ Absatz 1 Bundeskrebsregisterdatengesetz zum Jahreswechsel 2012/13 erfolgten Lieferungen der EKR an das Zentrum für Krebsregisterdaten extrahiert [23]. Zur Berechnung der Neuerkrankungszahlen wurden Daten von Registern eingeschlossen, deren Vollzähligkeit für die Gruppe der Leukämien nach ICD-10 C91-C95 für den Zeitraum 2001-2010 auf insgesamt über 90\%, für die Einzeljahre auf über $80 \%$ geschätzt wurde [21]. Der resultierende Pool umfasst Daten aus den EKR von SchleswigHolstein, Hamburg, Bremen, Mecklenburg-Vorpommern, Sachsen und Saarland für die Diagnosejahre 2001-2010. Die hämatoonkologischen Erkrankungsfälle wurden auf Basis der gelieferten oder konvertierten ICD-O-3-Morphologie-Kodes entsprechend der HAEMACARE-Gruppen zusammengefasst. Ausgewählt wurden anschließend alle Kategorien und Untergruppen lymphatischer und myeloischer Neubildungen, die Leukämien beinhalten, mit Ausnahme der sehr seltenen Burkitt-Zell- und der Plasmazellleukämien: chronische lymphatische Leukämien (CLL), reifzellige BZell-Leukämien, reifzellige T- und NKZell-Neoplasien, Vorläuferzellneoplasien einschließlich akuter lymphatischer Leukämien (ALL), lymphatische Leukämien ohne nähere Angaben, akute myeloische Leukämien (AML), myeloproliferative Neoplasien einschließlich chronischer myeloischer Leukämien (CML), myelodysplastische/myeloproliferative Neoplasien und unbekannte myeloische Neoplasien (• Tab. 1 und 2). Die Fallzahlen beziehen sich auf Personen ab einem Alter von 15 Jahren, für die altersstandardisierten Inzidenzraten wurden Kinder eingeschlossen. Für die Überlebenszeitanalysen in Bezug auf die 6 häufigsten spezifischen Erkrankungsgruppen wurden Daten aus denjenigen Krebsregistern verwendet, bei denen die Datenqualität entsprechend der aktuellen Ausgabe von „Krebs in Deutschland“ als ausreichend bewertet worden war [21]. Wesentliche Kriterien waren dabei ein Anteil von höchstens $15 \%$ betreffend nur über Todesbescheinigungen erfasste Fälle („death certificate only", DCO), sowie eine ausreichende Qualität des Vitalstatus-Follow-up. Letzteres gilt nach empirischen Untersuchungen als erfüllt, wenn das relative 5-Jahres-Überleben von Patienten mit Bauchspeicheldrüsen- bzw. metasta- siertem Lungenkrebs im Mittel bei maximal 7,5\% liegt [21, 24]. Die Überlebenszeitanalysen beruhen somit auf überwiegend leukämischen Erkrankungsfällen aus den Jahren 2003-2010 bei Patienten ab einem Alter von 15 Jahren aus Hamburg, Bremen, Niedersachsen, Mecklenburg-Vorpommern, Brandenburg, Saarland, Sachsen und Thüringen unter Ausschluss der DCO-Fälle. Diese 8 Bundesländer repräsentieren etwa $25 \%$ der deutschen Bevölkerung.

\section{Statistische Analysen}

Altersstandardisierte Inzidenzraten wurden pro 100.000 nach Kalenderjahr, Geschlecht und ausgewählten HAEMACARE-Erkrankungsgruppen berechnet, indem für jede 5-Jahres-Altersklasse die beobachteten Fälle einschließlich DCO pro 100.000 der durchschnittlichen Bezugsbevölkerung entsprechend der Europa-Standardbevölkerung gewichtet und aufsummiert wurden [IR (ASR Europa)]. Dargestellt sind die Mittelwerte der IR (ASR Europa) für den Zeitraum 2001 bis 2010 sowie für die häufigsten Erkrankungsgruppen die Zeittrends nach Diagnosejahr und Geschlecht.

Als Schätzer des krebsspezifischen Überlebens für 2008 bis 2010 diagnostizierte Erkrankungen wurde das relative 5-Jahres-Überleben nach dem Periodenansatz für jede der ausgewählten HAEMACARE-Gruppe insgesamt sowie nach Geschlecht und Altersgruppe ermittelt [25]. Das relative Überleben ist definiert als der Quotient aus dem beobachteten Überleben von Krebspatienten in einem bestimmten Zeitintervall und der im gleichen Intervall erwarteten Überlebensrate einer nach Alter, Geschlecht und Wohnregion entsprechenden Bevölkerungsgruppe [26]. Das erwartete Überleben wurde aus den alters-, geschlechts- und kalenderjahrspezifischen Sterbewahrscheinlichkeiten für die bundesdeutsche Bevölkerung entsprechend der Ederer-II-Methode abgeleitet [27, 28]. Für die Inzidenzberechnungen wurde das von OFFIS (Oldenburger Forschungs- und Entwicklungsinstitut für Informatik) entwickelte CARESS-Programm und für die Überlebenszeitanalysen periodR angewendet [29]. 


\section{Ergebnisse}

Die für die Inzidenzberechnungen ausgewählten Landeskrebsregister erfassen Krebserkrankungen von etwa 12 Mio. Einwohnern in 6 Bundesländern, entsprechend $15 \%$ der bundesdeutschen Bevölkerung. In - Tab. 1 und 2 sind die Fallzahlen für die insgesamt 11.292 lymphatischen und 12.036 myeloischen bösartigen Neubildungen bei Personen ab einem Alter von 15 Jahren im Zeitraum 2001 bis 2010 dargestellt. In den Listen finden sich die zu dem Oberbegriff „Leukämien“ "benannten Erkrankungsgruppen sowie die zugehörigen einzelnen ICDO-3-Morphologiekodes. Je Haupt- und Untergruppe sind die prozentualen $\mathrm{Ge}$ schlechts- und Altersgruppenverteilungen sowie als Datenqualitätsindikator die DCO-Anteile angegeben.

Absolut am häufigsten wurden chronische lymphatische Leukämien (CLL) mit einer Fallzahl von n=7937 erfasst, gefolgt von akuten myeloischen Leukämien (AML) $(\mathrm{n}=5277)$ und myeloproliferativen Neoplasien $(\mathrm{MPN})(\mathrm{n}=4311)$ einschließlich der chronischen myeloischen Leukämie (CML) ( $n=1523)$. Auf der Ebene der einzelnen Morphologiekodes zeigt sich an den Fallzahlen eine ungleichmäßige Verteilung und eine Bevorzugung von Begriffen mit dem Zusatz „ohne nähere Angabe“ (o.n.A.). Letztere betreffen, sofern vorhanden, jeweils etwa die Hälfte der Fälle in einer Kategorie. Absolut überwiegen bei den lymphatischen Leukämiegruppen in allen Gruppen und insgesamt die männlichen Patienten mit 50-70 bzw. $58 \%$, während sich das Geschlechterverhältnis bei den Fallzahlen der myeloischen Leukämien eher ausgewogen darstellt. 50\% und mehr der von CLL, AML und myelodysplastischen/myeloproliferativen Neoplasien betroffenen Patienten waren bei der Diagnose mindestens 70 Jahre alt. Im Gegensatz dazu überwiegen bei der Vorläuferzellneoplasie (ALL) die Erkrankungen der Jugendlichen und jüngeren Erwachsenen, wobei die noch häufigeren Fälle im Kindesalter unter 15 Jahren in dieser Betrachtung nicht berücksichtigt sind. Der Anteil von DCOFällen liegt für die spezifischen Leukämiegruppen zwischen 2 und 11\%. Leukämiefälle „ohne nähere Angabe“, d. h.

Bundesgesundheitsbl 2014·57:93-102 DOI 10.1007/s00103-013-1869-0

(c) Springer-Verlag Berlin Heidelberg 2013

\section{A. Nennecke · A. Wienecke · K. Kraywinkel Inzidenz und Überleben bei Leukämien in Deutschland nach aktuellen standardisierten Kategorien}

\section{Zusammenfassung}

Hintergrund. Leukämien umfassen eine Vielzahl unterschiedlicher Krebserkrankungen des blutbildenden Systems. Unbeständige Definitionen und Klassifikationen erschweren bevölkerungsbezogene Vergleiche und Aussagen zu zeitlichen Trends. 2010 wurde im Rahmen des europäischen HAEMACARE-Projekts eine aktuell für Epidemiologieund Public-Health-Zwecke nutzbare Gruppierung auf Basis der ICD-O-3 (Internationale Klassifikation der Krankheiten für die Onkologie, 3. Revision) und der 2008 veröffentlichten WHO-Klassifikation konsentiert. Erstmals werden für Deutschland nach diesem System Leukämieneuerkrankungen und -überleben anhand von Krebsregisterdaten differenziert dargestellt.

Material und Methoden. Aus dem Pool der Anfang 2013 von den epidemiologischen Landeskrebsregistern an das Robert Koch-Institut gelieferten Daten wurden Leukämiefälle für die Diagnosejahre 2001-2010 extrahiert und gemäß HAEMACARE gruppiert. Direkt altersstandardisierte Inzidenzraten wurden für den gesamten Zeitraum ermittelt. Zur
Schätzung der Prognose von 2008-2010 diagnostizierten erwachsenen Leukämiepatienten wurde das relative 5 -Jahres-Überleben nach dem Periodenansatz berechnet.

Ergebnisse und Diskussion. Ausgehend von 23.328 überwiegend leukämischen Erkrankungsfällen, dokumentiert für 6 Bundesländer über 10 Jahre, betragen die altersstandardisierten Inzidenzraten für chronische lymphatische Leukämien 4,1 (pro 100.000 Bevölkerung), gefolgt von akuten myeloischen Leukämien $(3,1)$ und myeloproliferativen Neubildungen $(2,4)$, ähnlich wie in anderen europäischen Regionen. Das relative 5-Jahres-Überleben liegt aktuell für Deutschland je nach Geschlecht, Erkrankungs- und Altersgruppe zwischen 8 und 95\%, für alle Leukämiearten damit deutlich über den für 20002002 anhand eines europäischen Datenpools ermittelten Werten.

Schlüisselwörter

Leukämien · Klassifikation · Krebsregister . Inzidenz · Überleben

\section{Leukemia incidence and survival in Germany according to current standardized categories}

\section{Abstract}

Background. Leukemias include a large number of diverse malignancies concerning the blood-forming system. Population-based comparisons and time trends are complicated by inconsistent definitions and classifications. In the course of the European HAEMACARE project, a consensus grouping system was created based on the morphology codes of the ICD-0-3 (International Classification of Diseases-Oncology, third revision), consistent with the WHO classification published in 2008 and useful for epidemiologic and public health purposes. For the first time leukemia incidence and survival in Germany are presented according to this system on the basis of cancer registry data..

Material and methods. A set of leukemia cases diagnosed during 2001-2010 was extracted from the data submitted by German population-based cancer registries to the Robert Koch Institute in early 2013 and grouped according to the HAEMACARE system. Direct age-standardized incidence rates were calculated. To estimate the prognosis of adult leukemia patients diagnosed during 2008-2010, the 5-year relative survival was computed using the period approach. Results and discussion. Based on 23,328 predominantly leukemic malignancies ascertained for six federal states over a 10-year period, the age-standardized incidence rates were 4.1 (per 100,000) for chronic lymphocytic leukemia, 3.1 for acute myeloid leukemia, and 2.4 for myeloproliferative neoplasms, similar to other European regions. The 5-year relative survival of leukemia in Germany currently ranges between 8 and $95 \%$ according to sex, age, and category, and is thus considerably better compared with results derived from a European data pool referring to 2000-2002.

\section{Keywords}

Leukemia - Classification - Cancer registry . Incidence · Survival 


\section{Leitthema}

Tab. 1 Lymphatische Leukämiegruppen nach HAEMACARE: Neuerkrankungsfälle nach Geschlecht und Alter, DCO-Anteile und altersstandardisierte Inzidenzraten (IR, Fälle pro 100.000 und Jahr, Europastandard), Datenpool aus 6 Landeskrebsregistern 2001 bis 2010

\begin{tabular}{|c|c|c|c|c|c|c|c|c|c|c|c|}
\hline \multirow{2}{*}{$\begin{array}{l}\text { Bezeichnung } \\
\text { der Erkran- } \\
\text { kungsgruppe }\end{array}$} & \multicolumn{2}{|l|}{ ICD-0-3 } & \multirow{2}{*}{$\begin{array}{l}\text { Fallzahl } \\
\text { (N) }\end{array}$} & \multirow{2}{*}{$\begin{array}{l}\text { Männer } \\
\text { (\%) }\end{array}$} & \multicolumn{3}{|c|}{ Altersverteilung (\%) } & \multirow{2}{*}{$\begin{array}{l}\mathrm{DCO}^{\mathrm{a}} \\
(\%)\end{array}$} & \multicolumn{3}{|c|}{ IR (ASR Europa) } \\
\hline & Klartext & $\begin{array}{l}\text { Morph.- } \\
\text { Kode }\end{array}$ & & & $15-49$ & $50-69$ & $70+$ & & Männer & Frauen & Gesamt \\
\hline \multicolumn{12}{|c|}{ Reifzellige (periphere) B-Zell-Neoplasien } \\
\hline \multicolumn{3}{|c|}{ Chronische lymphatische Leukämie (CLL) } & 7937 & 58,3 & 4,5 & 42,9 & 52,6 & 7,2 & 5,67 & 2,94 & 4,12 \\
\hline & $\begin{array}{l}\text { Chronische lymphatische } \\
\text { B-Zell-Leukämie/kleinzelliges } \\
\text { lymphozytisches Lymphom } \\
\text { (CLL/SLL) }\end{array}$ & $9823 / 3$ & 7297 & & & & & & & & \\
\hline & $\begin{array}{l}\text { Kleinzelliges lymphozytisches } \\
\text { B-Zell-Lymphom }\end{array}$ & $9670 / 3$ & 640 & & & & & & & & \\
\hline \multicolumn{3}{|c|}{ Reifzellige B-Zell-Leukämien } & 455 & 69,7 & 18,2 & 47,7 & 34,1 & 2,2 & 0,42 & 0,15 & 0,27 \\
\hline & $\begin{array}{l}\text { Prolymphozytenleukämie } \\
\text { vom B-Zell-Typ }\end{array}$ & $9833 / 3$ & 39 & & & & & & & & \\
\hline & Haarzellleukämie & $9940 / 3$ & 416 & & & & & & & & \\
\hline \multicolumn{3}{|c|}{ Reifzellige T-und NK-Zell-Neoplasien } & 1278 & 57,7 & 20,3 & 39,1 & 40,5 & 6,1 & 1,01 & 0,58 & 0,77 \\
\hline & $\begin{array}{l}\text { Reifzelliges T-Zell-Lymphom } \\
\text { o.n.A. }\end{array}$ & $9702 / 3$ & 564 & & & & & & & & \\
\hline & $\begin{array}{l}\text { Angioimmunoblastisches T- } \\
\text { Zell-Lymphom }\end{array}$ & $9705 / 3$ & 232 & & & & & & & & \\
\hline & $\begin{array}{l}\text { Großzelliges anaplastisches T- } \\
\text { Zell- und Null-Zell-Lymphom }\end{array}$ & $9714 / 3$ & 283 & & & & & & & & \\
\hline & $\begin{array}{l}\text { Hepatosplenisches Gamm- } \\
\text { Delta-Zell-Lymphom }\end{array}$ & $9716 / 3$ & 9 & & & & & & & & \\
\hline & Intestinales T-Zell-Lymphom & $9717 / 3$ & 28 & & & & & & & & \\
\hline & Aggressive NK-Zell-Leukämie & $9948 / 3$ & 11 & & & & & & & & \\
\hline & Nasales NK/T-Zell-Lymphom & $9719 / 3$ & 42 & & & & & & & & \\
\hline & $\begin{array}{l}\text { Adulte(s) T-Zell-Lymphom/ } \\
\text { Leukämie (HTLV1-positiv) }\end{array}$ & $9827 / 3$ & 50 & & & & & & & & \\
\hline & $\begin{array}{l}\text { Chronische lymphatische } \\
\text { T-Zell-Leukämie vom grobgra- } \\
\text { nulären Typ }\end{array}$ & $9831 / 3$ & 27 & & & & & & & & \\
\hline & $\begin{array}{l}\text { Prolymphozytenleukämie } \\
\text { vom T-Zell-Typ }\end{array}$ & $9834 / 3$ & 32 & & & & & & & & \\
\hline \multicolumn{3}{|c|}{$\begin{array}{l}\text { Vorläuferzellneoplasien [lymphoblastische Vorläuferzell- } \\
\text { lymphome/akute lymphatische Leukämien (ALL)] }\end{array}$} & 794 & 55,2 & 46,9 & 30,2 & 22,9 & 3,7 & 1,42 & 1,18 & 1,30 \\
\hline \multirow{3}{*}{$\begin{array}{l}\text { Lymphoblasti- } \\
\text { sche Vorläufer- } \\
\text { zelllymphome }\end{array}$} & $\begin{array}{l}\text { Lymphoblastisches Lymphom } \\
\text { der Vorläuferzellen }\end{array}$ & $9727 / 3$ & 39 & & & & & & & & \\
\hline & $\begin{array}{l}\text { B-lymphoblastisches Lym- } \\
\text { phom der Vorläuferzellen }\end{array}$ & $9728 / 3$ & 29 & & & & & & & & \\
\hline & $\begin{array}{l}\text { T-lymphoblastisches Lym- } \\
\text { phom der Vorläuferzellen }\end{array}$ & $9729 / 3$ & 27 & & & & & & & & \\
\hline \multirow{3}{*}{$\begin{array}{l}\text { Akute (Vorläufer- } \\
\text { zell-) lymphati- } \\
\text { sche Leukämien } \\
\text { (ALL) }\end{array}$} & $\begin{array}{l}\text { Vorläuferzell-lymphoblasti- } \\
\text { sche Leukämie o.n.A. }\end{array}$ & $9835 / 3$ & 398 & & & & & & & & \\
\hline & $\begin{array}{l}\text { Vorläuferzell-B-lymphoblasti- } \\
\text { sche Leukämie }\end{array}$ & $9836 / 3$ & 211 & & & & & & & & \\
\hline & $\begin{array}{l}\text { Vorläuferzell-T-lymphoblasti- } \\
\text { sche Leukämie }\end{array}$ & $9837 / 3$ & 90 & & & & & & & & \\
\hline \multicolumn{3}{|c|}{ Lymphatische Leukämien o.n.A. } & 828 & 50,1 & 4,0 & 18,5 & 77,5 & 72,5 & 0,56 & 0,31 & 0,41 \\
\hline & Lymphatische Leukämie o.n.A. & $9820 / 3$ & 815 & & & & & & & & \\
\hline & $\begin{array}{l}\text { Prolymphozytenleukämie } \\
\text { o.n.A. }\end{array}$ & $9832 / 3$ & 13 & & & & & & & & \\
\hline
\end{tabular}


Tab. 2 Myeloische Leukämiegruppen nach HAEMACARE: Neuerkrankungsfälle nach Geschlecht und Alter, DCO-Anteile und altersstandardisierte Inzidenzraten (IR, Fälle pro 100.000 und Jahr, Europastandard), Datenpool aus 6 Landeskrebsregistern 2001 bis 2010

\begin{tabular}{|c|c|c|c|c|c|c|c|c|c|c|c|}
\hline \multirow{2}{*}{$\begin{array}{l}\text { Bezeichnung } \\
\text { der Erkran- } \\
\text { kungsgruppe }\end{array}$} & \multicolumn{2}{|l|}{ ICD-0-3 } & \multirow{2}{*}{$\begin{array}{l}\text { Fallzahl } \\
\text { (N) }\end{array}$} & \multirow{2}{*}{$\begin{array}{l}\text { Männer } \\
\text { (\%) }\end{array}$} & \multicolumn{3}{|c|}{ Altersverteilung (\%) } & \multirow{2}{*}{$\begin{array}{l}\mathrm{DCO}^{\mathrm{a}} \\
(\%)\end{array}$} & \multicolumn{3}{|c|}{ IR (ASR Europa) } \\
\hline & Klartext & $\begin{array}{l}\text { Morph.- } \\
\text { Kode }\end{array}$ & & & $15-49$ & $50-69$ & $70+$ & & Männer & Frauen & Gesamt \\
\hline \multicolumn{3}{|c|}{ Akute myeloische Leukämie (AML) } & 5277 & 50,3 & 15,0 & 35,5 & 49,5 & 7,2 & 3,49 & 2,76 & 3,05 \\
\hline \multirow[t]{12}{*}{ AML, andere } & & & 4608 & 50,4 & 14,8 & 35,4 & 49,8 & 7,4 & 3,07 & 2,40 & 2,67 \\
\hline & $\begin{array}{l}\text { Akute myeloische Leukä- } \\
\text { mie, M6-Typ }\end{array}$ & $9840 / 3$ & 101 & & & & & & & & \\
\hline & $\begin{array}{l}\text { Akute myeloische Leukä- } \\
\text { mie o.n.A. }\end{array}$ & $9861 / 3$ & 2697 & & & & & & & & \\
\hline & $\begin{array}{l}\text { Akute myelomonozytäre } \\
\text { Leukämie }\end{array}$ & $9867 / 3$ & 467 & & & & & & & & \\
\hline & Akute Basophilenleukämie & $9870 / 3$ & 3 & & & & & & & & \\
\hline & $\begin{array}{l}\text { Akute myeloische Leuk- } \\
\text { ämie mit minimaler Aus- } \\
\text { reifung }\end{array}$ & $9872 / 3$ & 138 & & & & & & & & \\
\hline & $\begin{array}{l}\text { Akute myeloische Leukä- } \\
\text { mie ohne Ausreifung }\end{array}$ & $9873 / 3$ & 343 & & & & & & & & \\
\hline & $\begin{array}{l}\text { Akute myeloische Leukä- } \\
\text { mie mit Ausreifung }\end{array}$ & $9874 / 3$ & 406 & & & & & & & & \\
\hline & $\begin{array}{l}\text { Akute Monozytenleu- } \\
\text { kämie }\end{array}$ & $9891 / 3$ & 342 & & & & & & & & \\
\hline & $\begin{array}{l}\text { Akute Megakaryoblasten- } \\
\text { leukämie }\end{array}$ & $9910 / 3$ & 55 & & & & & & & & \\
\hline & Myelosarkom & $9930 / 3$ & 27 & & & & & & & & \\
\hline & $\begin{array}{l}\text { Akute Panmyelose mit } \\
\text { Myelofibrose }\end{array}$ & $9931 / 3$ & 29 & & & & & & & & \\
\hline \multicolumn{3}{|c|}{ AML mit zytogenetischen Anomalien } & 278 & 47,5 & 34,2 & 40,6 & 25,2 & 1,8 & 0,19 & 0,20 & 0,19 \\
\hline & $\begin{array}{l}\text { Akute Promyelozytenleu- } \\
\text { kämie, } t(15 ; 17) \\
\text { (q22;q11-12) }\end{array}$ & $9866 / 3$ & 152 & & & & & & & & \\
\hline & $\begin{array}{l}\text { Akute myelomonozytäre } \\
\text { Leukämie mit Eosinophilie }\end{array}$ & $9871 / 3$ & 48 & & & & & & & & \\
\hline & $\begin{array}{l}\text { Akute myeloische Leukä- } \\
\text { mie, } t(8 ; 21)(q 22 ; q 22)\end{array}$ & $9896 / 3$ & 71 & & & & & & & & \\
\hline & $\begin{array}{l}\text { Akute myeloische Leu- } \\
\text { kämie mit 11q23-Abnor- } \\
\text { mitäten }\end{array}$ & $9897 / 3$ & 7 & & & & & & & & \\
\hline \multicolumn{3}{|c|}{ AML mit Dysplasie mehrerer Zelllinien } & 365 & 50,7 & 3,3 & 31,5 & 65,2 & 8,8 & 0,22 & 0,15 & 0,18 \\
\hline & $\begin{array}{l}\text { Akute myeloische Leukä- } \\
\text { mie mit Dysplasie mehre- } \\
\text { rer Zelllinien }\end{array}$ & $9895 / 3$ & 324 & & & & & & & & \\
\hline & $\begin{array}{l}\text { Refraktäre Anämie mit } \\
\text { Blastenüberschuss in } \\
\text { Transformation [obs.] }\end{array}$ & $9984 / 3$ & 41 & & & & & & & & \\
\hline \multicolumn{3}{|c|}{ AML, therapiebedingt } & 26 & 50 & 19,2 & 53,8 & 26,9 & 7,7 & 0,02 & 0,02 & 0,02 \\
\hline & $\begin{array}{l}\text { Akute myeloische Leukä- } \\
\text { mie infolge Therapie o.n.A. }\end{array}$ & $9920 / 3$ & 20 & & & & & & & & \\
\hline & $\begin{array}{l}\text { Therapiebedingtes myelo- } \\
\text { dysplastisches Syndrom } \\
\text { o.n.A. }\end{array}$ & $9987 / 3$ & 6 & & & & & & & & \\
\hline \multicolumn{3}{|c|}{ Myeloproliferative Neoplasien } & 4311 & 48,6 & 15,4 & 38,0 & 46,5 & 8,0 & 2,71 & 2,19 & 2,41 \\
\hline \multicolumn{3}{|c|}{ Chronische myeloische Leukämie (CML) } & 1523 & 53,8 & 20,8 & 34,8 & 44,4 & 10,9 & 1,10 & 0,73 & 0,89 \\
\hline & $\begin{array}{l}\text { Chronische myeloische } \\
\text { Leukämie }\end{array}$ & $9863 / 3$ & 1263 & & & & & & & & \\
\hline & $\begin{array}{l}\text { Chronische myeloische } \\
\text { Leukämie, BCR/ABL positiv }\end{array}$ & $9875 / 3$ & 260 & & & & & & & & \\
\hline
\end{tabular}


Tab. 2 Myeloische Leukämiegruppen nach HAEMACARE: Neuerkrankungsfälle nach Geschlecht und Alter, DCO-Anteile und altersstandardisierte Inzidenzraten (IR, Fälle pro 100.000 und Jahr, Europastandard), Datenpool aus 6 Landeskrebsregistern 2001 bis 2010 (Fortsetzung)

\begin{tabular}{|c|c|c|c|c|c|c|c|c|c|c|c|}
\hline \multirow{2}{*}{$\begin{array}{l}\text { Bezeichnung } \\
\text { der Erkran- } \\
\text { kungsgruppe }\end{array}$} & \multicolumn{2}{|l|}{ ICD-0-3 } & \multirow{2}{*}{$\begin{array}{l}\text { Fallzahl } \\
\text { (N) }\end{array}$} & \multirow{2}{*}{$\begin{array}{l}\text { Männer } \\
\text { (\%) }\end{array}$} & \multicolumn{3}{|c|}{ Altersverteilung (\%) } & \multirow{2}{*}{$\begin{array}{l}\mathrm{DCO}^{\mathrm{a}} \\
(\%)\end{array}$} & \multicolumn{3}{|c|}{ IR (ASR Europa) } \\
\hline & Klartext & $\begin{array}{l}\text { Morph.- } \\
\text { Kode }\end{array}$ & & & $15-49$ & $50-69$ & $70+$ & & Männer & Frauen & Gesamt \\
\hline \multicolumn{3}{|c|}{ Andere myeloproliferative Neoplasien } & 2788 & 45,8 & 12,5 & 39,8 & 47,7 & 6,3 & 1,60 & 1,46 & 1,53 \\
\hline & Mastzellsarkom o.n.A. & $9740 / 3$ & 3 & & & & & & & & \\
\hline & Maligne Mastozytose & $9741 / 3$ & 58 & & & & & & & & \\
\hline & Mastzellleukämie & $9742 / 3$ & 2 & & & & & & & & \\
\hline & Polycythaemia vera & $9950 / 3$ & 815 & & & & & & & & \\
\hline & $\begin{array}{l}\text { Myelosklerose mit myeloi- } \\
\text { der Metaplasie }\end{array}$ & $9961 / 3$ & 647 & & & & & & & & \\
\hline & $\begin{array}{l}\text { Essenzielle Thrombozy- } \\
\text { thämie }\end{array}$ & $9962 / 3$ & 839 & & & & & & & & \\
\hline & $\begin{array}{l}\text { Chronische Neutrophilen- } \\
\text { leukämie }\end{array}$ & $9963 / 3$ & 6 & & & & & & & & \\
\hline & $\begin{array}{l}\text { Hypereosinophilie- } \\
\text { Syndrom }\end{array}$ & $9964 / 4$ & 9 & & & & & & & & \\
\hline & $\begin{array}{l}\text { Chronische myeloprolife- } \\
\text { rative Erkrankung (CMPE) } \\
\text { o.n.A. }\end{array}$ & $9960 / 3$ & 409 & & & & & & & & \\
\hline \multicolumn{3}{|c|}{ Myelodysplastische/myeloproliferative Neoplasien } & 734 & 55,7 & 4,8 & 29,8 & 65,4 & 9,4 & 0,50 & 0,28 & 0,37 \\
\hline & $\begin{array}{l}\text { Chronische myelomono- } \\
\text { zytäre Leukämie o.n.A. }\end{array}$ & $9945 / 3$ & 430 & & & & & & & & \\
\hline & $\begin{array}{l}\text { Atypische chronische mye- } \\
\text { loische Leukämie, BCR/ } \\
\text { ABL negativ }\end{array}$ & $9876 / 3$ & 47 & & & & & & & & \\
\hline & $\begin{array}{l}\text { Juvenile myelomonozytä- } \\
\text { re Leukämie }\end{array}$ & $9946 / 3$ & 0 & & & & & & & & \\
\hline & $\begin{array}{l}\text { Myeloproliferative Krank- } \\
\text { heit o.n.A. }\end{array}$ & $9975 / 1$ & 257 & & & & & & & & \\
\hline \multicolumn{3}{|c|}{ Unbekannte myeloide Neoplasien } & 1714 & 46,0 & 4,7 & 17,8 & 77,5 & 72,3 & 1,00 & 0,66 & 0,79 \\
\hline \multirow{3}{*}{$\begin{array}{l}\text { Leukämien } \\
\text { o.n.A. }\end{array}$} & Leukämie o.n.A. & $9800 / 3$ & 705 & & & & & & & & \\
\hline & Akute Leukämie o.n.A. & $9801 / 3$ & 188 & & & & & & & & \\
\hline & $\begin{array}{l}\text { Akute biphänotypische } \\
\text { Leukämie }\end{array}$ & $9805 / 3$ & 16 & & & & & & & & \\
\hline $\begin{array}{l}\text { Myeloische } \\
\text { Leukämie o.n.A. }\end{array}$ & $\begin{array}{l}\text { Myeloische Leukämie } \\
\text { o.n.A. }\end{array}$ & $9860 / 3$ & 805 & & & & & & & & \\
\hline
\end{tabular}

dokumentiert mit unspezifischen Morphologiekodes, machen bezogen auf die Gesamtfallzahl $11 \%$ aus, wobei der hohe DCO-Anteile von $72 \%$ und die im Vergleich zu den spezifischen Gruppen mit $78 \%$ überrepräsentierte höchste Altersklasse auffallen.

Die in den letzten 3 Spalten von - Tab. 1 und 2 präsentierten mittleren altersstandardisierten Inzidenzraten liegen je spezifischer Hauptgruppe und Geschlecht zwischen 5,7 pro 100.000 Europa-Standardbevölkerung für männliche CLL-Patienten und 0,02 für Patienten mit therapiebedingter AML. Durchgehend finden sich für Männer höhere IR (ASR Europa), wobei die geschlech- terspezifischen Differenzen mit Ausnahme der CLL weniger als 1/100.000 betragen. - Abb. 1 illustriert die Zeittrends bei den häufigsten Leukämiegruppen anhand der IR (ASR Europa) nach Geschlecht und Jahr. Insgesamt zeigen sich für Männer höhere Erkrankungsraten, besonders bei den CLL mit 4,7 bis 6,2 pro 100.000 gegenüber 2,4 bis 3,3 bei Frauen. Die Trends erscheinen über den beobachteten Zeitraum im Wesentlichen gleichbleibend mit Schwankungen um maximal 1,5/100.000, wobei die IR (ASR Europa) für das letzte Beobachtungsjahr tendenziell am niedrigsten liegen.

Das für die Kalenderperiode 2008 bis 2010 berechnete relative 5-Jahres-Über- leben von Erwachsenen ist in $\bullet$ Abb. 2 dargestellt, bezogen auf die jeweilige Erkrankungsgruppe insgesamt liegt es bei CLL mit 76,3\% am höchsten, bei AML mit 24,1\% am niedrigsten. Außer bei der ALL überleben weibliche Betroffene tendenziell etwas besser als Männer. Für alle Leukämieformen ist eine deutliche Verschlechterung der Prognose mit zunehmendem Alter erkennbar, das relative 5-Jahres-Überleben ist bei über 70-Jährigen oft nur halb so hoch wie bei den jungen Erwachsenen (15 bis 40 Jahre). 

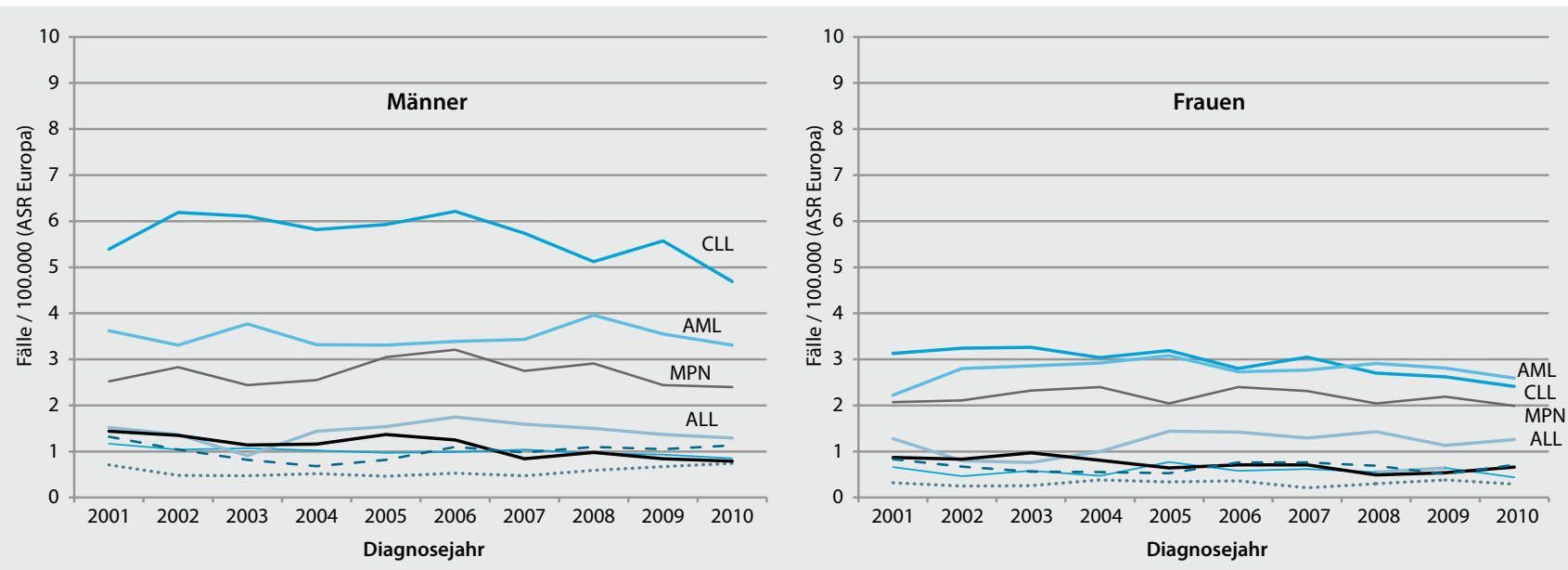

\begin{tabular}{|llll|}
\hline- Chronische lymphatische Leukämien (CLL) & - Reifzellige T- und NK-Zell-Neoplasien & Vorläuferzellneoplasien (ALL) \\
$\cdots \cdots . .$. & Lymphatische Leukämien o.n.A. & - Akute myeloische Leukämie (AML) & Myeloproliferative Neubildungen (MPN) \\
- Chronisch myeloische Leukämie (CML) & -- & Unbekannte myeloide Neoplasien
\end{tabular}

Abb. $1 \Delta$ Zeittrends bei den häufigsten Leukämieerkrankungsgruppen in Deutschland: altersstandardisierte Inzidenzraten nach Geschlecht und Kalenderjahr pro 100.000 (Europastandard), Datenpool aus 6 Landeskrebsregistern 2001 bis 2010

\section{Diskussion}

Inzidenz und Überleben gehören zu den wichtigsten Parametern, um die Krankheitslast in Bevölkerungen zu quantifizieren. Für Leukämien wurden diesbezügliche Aussagen in großen epidemiologischen Analysen in der Vergangenheit oft nur nach groben Kategorien getroffen, etwa Leukämien insgesamt oder unterteilt nach den Attributen „akut" vs. „chronisch" und "lymphatisch" vs. „myeloisch" $[4,30,31]$. Inzwischen stellen mehrere epidemiologische Landeskrebsregister in Deutschland Neuerkrankungs- und Sterblichkeitszahlen deutlich differenzierter über webbasierte Datenbanken öffentlich zur Verfügung und ermöglichen so eine gute Orientierung über das Krebsgeschehen [19]. Die hier meist zur Klassifikation der bösartigen Neubildungen verwendete ICD-10 begrenzt die unmittelbare Nutzung dieser Daten für die Klinik und Wissenschaft in Bezug auf leukämische Erkrankungen, denn die ICD10 ist aufgrund der unterschiedlichen Struktur und Aktualität mit den vor allem im klinischen Bereich eingesetzten WHO „Blue Books" und der ICD-O-3 nur bedingt vereinbar $[6,7,8,17]$. Durch die Nutzung der in den Krebsregisterdatenbeständen regelhaft dokumentierten ICD-O-3-Morphologiekodes in Ver- bindung mit dem oben beschriebenen HAEMACARE-Gruppierungssystem ist es jedoch möglich, epidemiologische Aussagen zu Leukämien in Deutschland unter Berücksichtigung der aktuellen WHO-Klassifikationen abzuleiten.

\section{Neuerkrankungen}

Die so für den Zeitraum 2001 bis 2010 ermittelten deutschen Inzidenzraten leukämischer Erkrankungen (• Tab. 1 und 2) liegen auf ähnlichem Niveau wie die für 2000 bis 2002 für den europäischen Raum veröffentlichten Ergebnisse, wobei die Vergleichbarkeit eingeschränkt ist durch die Angabe altersstandardisierter Raten nach der realen Europabevölkerung bei HAEMACARE und die Gewichtung nach europäischer Standardbevölkerung in der vorliegenden Arbeit [3]. Generell sind Männer häufiger als Frauen von Leukämien betroffen; am deutlichsten zeigt sich dies bei der insgesamt am häufigsten auftretenden CLL. Die geschlechtsspezifische Differenz ist ein bekanntes Phänomen bei hämatoonkologischen Erkrankungen, möglicherweise ist sie zurückzuführen auf eine geringere Exposition von Frauen gegenüber umwelt- und arbeitsbedingten Risikofaktoren oder auch auf hormonelle und genetische Faktoren [3, 32]. Mit Ausnahme der ALL betreffen Leukämien überwiegend ältere Menschen, 50\% der Fälle und mehr entfallen bei den hier untersuchten Erkrankungsgruppen auf Patienten im Alter ab 70 Jahren. Die mit 78\% deutlich höheren Anteile dieser Altersgruppe an den als unspezifisch dokumentierten Leukämien könnten auf eine schlechtere diagnostische Aufarbeitung, aber auch auf höhere Anteile an nicht differenziert dokumentierbaren DCO-Fällen hinweisen. Die aufgelisteten Fallzahlen für die einzelnen Morphologietypen erlauben eine grobe Einschätzung der Häufigkeiten, sie zeigen allerdings eine Tendenz zu allgemeineren, weniger spezifischen Bezeichnungen. Inwieweit diese Verteilungen der realen Situation diagnostischer Aussagen entsprechen oder eher artifiziell durch Fehler und Ungenauigkeiten bei der Klassifikation, Erfassung, Kodierung und Konversion beispielsweise von der ICD-O-2 zur ICD-O-3 bedingt sind, lässt sich derzeit nicht beurteilen [18]. Die in - Abb. 1 präsentierten Zeittrends der altersstandardisierten Neuerkrankungsraten lassen keine auffälligen Auf- oder Abwärtsentwicklungen erkennen; dies spricht ebenso wie die mit den Europadaten von 2000 bis 2002 vergleichbaren Niveaus für eine stabile Erfassung. Die leichte Tendenz zu niedrigeren Werten am Ende des Beobachtungs- 


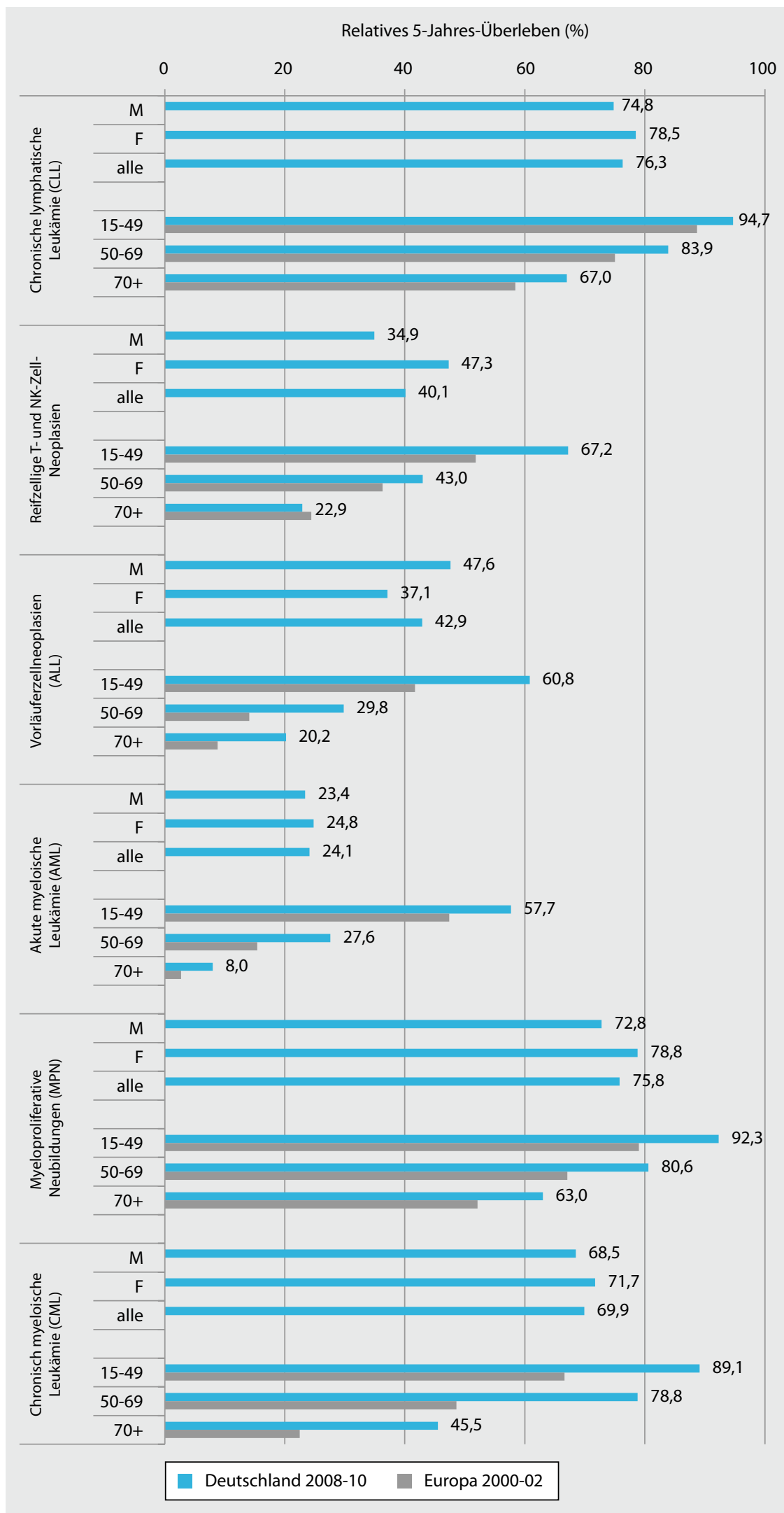

Abb. $2 \Delta$ Relatives 5-Jahres-Überleben für die häufigsten Leukämieerkrankungsgruppen in Deutschland nach Geschlecht und Altersgruppen, Datenpool aus 8 Landeskrebsregistern, Periode 2008 bis 2010; zum Vergleich altersgruppenspezifisches relatives 5-Jahres-Überleben in Europa, Periode 2000 bis 2002. (Nach $[9,35])$ zeitraums lässt sich am ehesten mit der zu vermutenden Zeitverzögerung zwischen Diagnosestellung und Meldung erklären, d. h., für einen Teil der 2009 und 2010 inzidenten Fälle sind noch verspätete Meldungen zu erwarten.

\section{Überleben}

Das Überleben bei Leukämieerkrankungen weist eine große Variationsbreite auf. Mit 76\% relativem 5-Jahres-Überleben haben CLL-Patienten vergleichsweise gute Prognosen im Gegensatz zu AMLPatienten mit 24\%. Das tendenziell etwas bessere Überleben von Frauen mit hämatoonkologischen Erkrankungen wurde schon häufiger beschrieben [33, 34]. Hingegen finden sich für den hier bei der ALL auffälligen Überlebensvorteil männlicher Patienten keine analogen Ergebnisse in der Literatur; er zeigt sich stabil bei stratifizierten Analysen nach Regionen und Zeitperioden (Ergebnisse nicht dargestellt). Zum orientierenden Vergleich sind in $\mathbf{A b b} .2$ die im Rahmen des HAEMACARE-Projektes für Europa und 2000 bis 2002 ermittelten altersgruppenspezifischen Ergebnisse als hellgraue Balken dargestellt $[9,35]$. Demnach erscheint das für deutsche Leukämiepatienten und 2008 bis 2010 berechnete Überleben über alle Leukämieerkrankungsgruppen deutlich verbessert: Die Differenzen des relativen 5-Jahres-Überlebens betragen bei CML 22 bis 30 Prozentpunkte, bei CLL, ALL, AML und myeloproliferativen Neoplasien 5 bis 19 Prozentpunkte. Die deutlichen Steigerungen bei CML sind vermutlich durch die um die Jahrtausendwende etablierte sog. zielgerichtete Therapie mit Imatinib zu erklären [36]. Die trotz der insgesamt zu beobachtenden Fortschritte weiter bestehende ausgeprägte Altersabhängigkeit der Prognose könnte sowohl auf eine verzögerte Diagnostik, Versorgungsnachteile und mangelnde Forschungs- und Leitlinienarbeit betreffend höhere Altersgruppen hinweisen als auch ein Indiz für eine häufigere und schwerwiegendere Komorbidität und eine schlechtere Therapietoleranz sein [37]. 


\section{Datenqualität}

In Bezug auf die Qualität weisen die hier genutzten Daten mit 14\% DCO-Fällen auf eine noch relevante Untererfassung der inzidenten Fälle zu Lebzeiten der Patienten hin, die zumindest für die jüngeren Register auf die während der frühen 2000er-Jahre noch kurze Laufzeit zurückzuführen ist. $11 \%$ der eingeschlossenen Fälle ( $n=2542)$ wurden mittels Morphologiekodes für explizit unspezifische Leukämiegruppen (lymphatische Leukämien o.n.A, unbekannte myeloische Neoplasien) dokumentiert; im Vergleich zu den von Sant et al. für den europäischen Raum Anfang des Jahrtausends ermittelten $18 \%$ eine akzeptable, jedoch noch optimierbare Größenordnung [3]. Geht man von der Zuordnung der Leukämien nach ICD-10 (C91-C95) aus, so ist die Zahl der Fälle mit fehlender oder komplett unspezifischer Histologie (ICD-O-3 $8000)$ vergleichsweise gering $(\mathrm{n}=148)$. Umgekehrt waren deutlich mehr der in der vorliegenden Untersuchung eingeschlossenen Fälle $(n=4973)$ nach ICD10 den Lymphomen (C82-C85) oder den Neubildungen unsicheren oder unbekannten Verhaltens des blutbildenden Systems (D45-D47) zugeordnet, die nach HAEMACARE als Leukämien aufgefasst werden. Dieser Sachverhalt erklärt die resultierenden deutlich höheren Inzidenzraten für Leukämien im Verhältnis zu den sonst üblichen bevölkerungsbezogenen Auswertungen nach ICD-10.

\section{Fazit}

Die Stärken dieser Untersuchung liegen in der Größe der Datenbasis und der damit anzunehmenden Repräsentativität für das Leukämiegeschehen in Deutschland aus epidemiologischer Perspektive sowie in der Aktualität der Daten und der angewandten Klassifikationen. Zum ersten Mal werden differenzierte Zahlen zu Leukämien in der deutschen Bevölkerung dargestellt, die auf den morphologischen Zelltypbezeichnungen der ICD-0-3 basieren und die medizinischwissenschaftlich anerkannten Klassifikationen der WHO berücksichtigen. Damit sowie mit dem Gegenwartsbezug des ausgewählten Diagnosezeitraums wird es möglich, die Ergebnisse in Relation zu aktuellen klinischen Daten nach den WHO „Blue Books“ zu setzen. Ebenso können die Inzidenzraten und das relative 5-Jahres-Überleben für internationale Vergleiche nach den HAEMACARE-Gruppen verwendet werden. Unsere Arbeit zeigt exemplarisch die mögliche Verbindung von Public-Health-Informationen und klinischer Forschung auf.

Trotz der beschriebenen Schwächen der Datenqualität und des Verbesserungsbedarfs der Krebsregistrierung liefert diese Übersicht somit eine gute und auch für den klinisch-wissenschaftlichen Bereich nutzbare Orientierung zu Häufigkeiten und Prognosen leukämischer Erkrankungen.

\section{Korrespondenzadresse}

\section{Dr. A. Nennecke}

Hamburgisches Krebsregister, Behörde für Gesundheit und Verbraucherschutz (BGV) Billstr. 80, 20539 Hamburg Alice.Nennecke@bgv.hamburg.de

Danksagungen. Wir danken dem Bremer Krebsregister, dem Hamburgischen Krebsregister, dem Epidemiologischen Krebsregister Niedersachsen, dem Krebsregister Schleswig-Holstein, dem Epidemiologischen Krebsregister Saarland und dem Gemeinsamen Krebsregister der neuen Bundesländer und Berlins für die Datenbereitstellung.

\section{Einhaltung ethischer Richtlinien}

Interessenkonflikt. A. Nennecke, A. Wienecke und K. Kraywinkel geben an, dass kein Interessenkonflikt besteht.

Dieser Beitrag beinhaltet keine Studien an Menschen oder Tieren.

\section{Literatur}

1. Piller G (2001) Leukaemia - a brief historical review from ancient times to. Br J Haematol 112:282-292

2. Naegeli O (1900) Über rothes Knochenmark und Myeloblasten. Dtsch Med Wochenschr 26:287

3. Sant M, Allemani C, Tereanu C et al (2010) Incidence of hematologic malignancies in Europe by morphologic subtype: results of the HAEMACARE project. Blood 116(19):3724-3734 [Erratum in: Blood 117(12):3477]

4. Curado MP, Edwards B, Shin HR (2007) Cancer incidence in five continents, Vol. IX. IARC Scientific Publications No. 160, Lyon
5. HAEMACARE - Cancer Registry Based project on haematological malignancies: background, rationale and aims [Internet]. http://www.haemacare. eu/project.asp (Zugegriffen: 14. Mai 2013)

6. Fritz A, Percy C, Shanmugaratnam K et al (Hrsg) (2000) International classification of diseases for oncology (ICD-O), 3. Aufl. World Health Organization, Geneva

7. Jaffe ES, Harris LN, Stein H et al (2001) World Health Organization classification of tumours: pathology and genetics of tumours of haematopoietic and lymphoid Tissues. IARC Press, Lyon

8. Swerdlow SH, Campo E, Harris NL et al (2008) World Health Organization classification of tumours of haematopoietic and lymphoid tissues. IARC Press, Lyon

9. Marcos-Gragera R, Allemani C, Tereanu C et al (2011) Survival of European patients diagnosed with lymphoid neoplasms in 2000-2002: results of the HAEMACARE project. Haematologica 96(5):720-728

10. HAEMACARE Working Group (2010) Manual for coding and reporting haematological malignancies. Tumori 96(4):i-A32

11. Interaktive Datenbank des Bremer Krebsregisters. http://www.krebsregister.bremen.de/datenbank. php (Zugegriffen: Mai 2013)

12. Interaktive Datenbank des Hamburgischen Krebsregisters. http://www.krebsregister-hamburg.de/ (Zugegriffen: Mai 2013)

13. Interaktive Datenbank des epidemiologischen Krebsregisters Niedersachsen. http://www.krebsregister-niedersachsen.de/registerstelle/?page_ id=74 (Zugegriffen: Mai 2013)

14. Interaktive Datenbank des epidemiologischen Krebsregisters NRW gGmbH. http://www.krebsregister.nrw.de/index.php?id=113 (Zugegriffen: Mai 2013)

15. Interaktive Datenbank des epidemiologischen Krebsregisters Saarland. http://www.krebsregister.saarland.de/datenbank/datenbank.html (Zugegriffen: Mai 2013)

16. Interaktive Datenbank des Krebsregisters Schleswig-Holstein. http://www.krebsregister-sh.de/datenbank/index.html (Zugegriffen: Mai 2013)

17. DIMDI (Deutsches Institut für Medizinische Dokumentation und Information) (2010) Kommentar zur ICD-10-GM Version 2011 [Internet]. http:// www.dimdi.de/static/de/klassi/icd-10-gm/kodesuche/onlinefassungen/htmlgm2011/zusatzkommentar.htm (Zugegriffen: 14. Mai 2013)

18. Kraywinkel K, Altmann U, Holzmann M et al (2013) Diagnosekodierung nach ICD-O-3 und ICD10 in epidemiologischen und klinischen Krebsregistern. mdi - Forum der Medizin_Dokumentation und Medizin_Informatik 2:52-56

19. Gesellschaft der epidemiologischen Krebsregister in Deutschland e.V. Atlas der Krebsinzidenz und -mortalität in Deutschland (GEKID-Atlas). Datenlieferung: Dezember 2012, Lübeck, 2013. http:// www.gekid.de (Zugegriffen: Mai 2013)

20. Robert Koch-Institut (Hrsg) (o J) Datenbankabfrage Zentrum für Krebsregisterdaten. http://www. krebsdaten.de/Krebs/DE/Home/Datenbankabfrage/datensaetze_gesamt_tabelle.html (Zugegriffen: Mai 2013)

21. Robert Koch-Institut, die Gesellschaft der epidemiologischen Krebsregister in Deutschland e.V. (Hrsg) (2012) Krebs in Deutschland 2007/2008. 8. Ausgabe. Berlin 


\section{Leitthema}

22. Hiripi E, Gondos A, Emrich K et al (2012) Survival from common and rare cancers in Germany in the early 21st century. Ann Oncol 23(2):472-479

23. Bundeskrebsregisterdatengesetz vom 10 . August 2009, BGBI. I S. 2707

24. Nennecke A, Barnes B, Brenner $H$ et al (2012) Datenqualität oder Unterschiede in der onkologischen Versorgung? - Berichtsstandards für Überlebenszeitanalysen mit Krebsregisterdaten. Gesundheitswesen 74:1-5

25. Brenner H, Gefeller O (1996) An alternative approach to monitoring cancer patient survival. Cancer 78:2004-2010

26. Ederer F, Axtell LM, Cutler SJ (1961) The relative survival rate: a statistical methodology. Natl Cancer Inst Monogr 6:101-121

27. Ederer F, Heise $H$ (1959) Instructions to IBM 650 programmers in processing survival computations. Bethesda (MD) Methodological note No. 10, End Results Evaluation Section, National Cancer Institute, Bethesda MD

28. Hakulinen T, Seppä K, Lambert PC (2011) Choosing the relative survival method for cancer survival estimation. Eur J Cancer 47:2202-2210

29. Holleczek B, Gondos A, Brenner H (2009) periodR - an $R$ package to calculate long-term cancer survival estimates using period analysis. Methods Inf Med 48:123-128

30. Ferlay J, Shin HR, Bray F et al (2010) GLOBOCAN 2008 v2.0, Cancer Incidence and Mortality Worldwide: IARC CancerBase No. 10 [Internet]. International Agency for Research on Cancer, Lyon. http://globocan.iarc.fr (Zugegriffen: 14. Mai 2013)

31. Verdecchia A, Santaquilani M, Sant M (2009) Survival for cancer patients in Europe. Ann Ist Super Sanita 45(3):315-324

32. Cook MB, Dawsey SM, Freedman ND et al (2009) Sex disparities in cancer incidence by period and age. Cancer Epidemiol Biomarkers Prev 18(4):1174-1182

33. Monnereau A, Troussard X, Belot A et al (2013) Unbiased estimates of long-term net survival of hematological malignancy patients detailed by major subtypes in France. Int J Cancer 132(10):23782387

34. Broek EC van den, Kater AP, Schans SA van de (2012) Chronic lymphocytic leukaemia in the Netherlands: trends in incidence, treatment and survival, 1989-2008. Eur J Cancer 48(6):889-895

35. Maynadié M, De Angelis R, Marcos-Gragera R et al (2013) Survival of European patients diagnosed with myeloid malignancies: a HAEMACARE study. Haematologica 98(2):230-238

36. Hehlmann R, Hochhaus A, Baccarani M (2007) Chronic myeloid leukaemia. Lancet 370:342-350

37. Quaglia A, Tavill A, Shack L et al (2009) The cancer survival gap between elderly and middleaged patients in Europe is widening. Eur $\mathrm{J}$ Cancer 45(6):1006-1016 